\title{
Estimation of frequency characteristics of photodiode determined by motion of charge carriers in the space-charge region
}

\author{
A.I. Danilyuk, Yu.G. Dobrovolskiy \\ Tensor ltd, 226, Chervonoarmiyska str., 58013 Chernivtsi, Ukraine \\ E-mail:chtenz@chv.ukrpack.net
}

\begin{abstract}
Frequency characteristics of photodiode determined by motion of charge carriers in the space-charge region was estimated. It was shown that the current in the external circuit depends on two functions (their specific kind) of coordinates of the electric field and generation density of photodetector current.
\end{abstract}

Keywords: frequency characteristics, photodetector, charge carrier, space-charge region.

Manuscript received 17.03.06; accepted for publication 23.10.06.

\section{Introduction}

Characteristics of each photodiode, as a basic structure of photodetector, in the general case are determined by construction of photodiode. In particular, these are characteristics of the used material, configuration of electric fields, mobilities of charge carriers, width of the space-charge region (SCR). In addition, characteristics of photodiode are determined by the external applied voltage and wave length of the used optical radiation. In the case of its absorption, only in its SCR and at negligibly small distances around it, for example, in a $p-i$ - $n$ photodiode, frequency characteristics are determined, mainly, by the time-of-flight of the generated charge carriers through the SCR [1-4].

As the frequency characteristics of photodiode are one of basic, determining quality and possibilities of every specific photodiode, the task to exactly estimate these characteristics becomes actual for the modern electronic technique, in particular, for constructing the semiconductor photodiodes.

In $[1,5]$, the problem of kinetics of the photodiode response related to the flight of charge carriers through SCR was considered. However, this consideration has not enough deep character, what does not allow to use its results for engineering computations when constructing the high-frequency photodiodes. In [6], considered were the dependence of photodiode output signal and its frequency characteristics on the input resistance and capacity of preamplifier. This research and that of the influence of explosive noise on the parameters of a photodiode performed in [7] cover the specific questions related to constructing the photodiode.

\section{General case of drift of charge carriers inside the semiconductor crystal in the electric field of SCR}

We will consider a few extreme cases, when frequency characteristics are determined by only the time-of-flight of charge carriers through the SCR. For simplicity of consideration, we will consider a "flat" SCR, in which the vector of the electric field is directed along the axis $\vec{x}$, i.e., the case of the "flat electric field" shown in the figure.

$$
\vec{E}(\vec{x}, \vec{y}, \vec{z}) \equiv \vec{E}_{x}(x)=\vec{i} E_{x},
$$

$E_{y}=E_{z}=0$,

where $E_{x}, E_{y}, E_{z}$ are the components of the electric field vector; $\vec{x}, \vec{y}, \vec{z}$ are the basis vectors (axes) of the chosen rectangular (Cartesian) co-ordinates in a threedimensional space; $\vec{i}$ is the unit vector directed along the axis $\vec{x}$.

Let one (initial) boundary of SCR coincides with the coordinate plane $\{\vec{y}, \vec{z}\}$, and the second (final) one with the plane parallel to it passing through a point with the coordinate $x=x_{0}$. The accepted terms are allowed, not violating community of consideration on the whole, to consider a motion of charge carriers only along the axis $\vec{x}$. We will choose inside the SCR two elementary layers as flat regions parallel to the SCR scope with the thicknesses of $d x$ and $d x^{\prime}$ and passing in the vicinity of points with the $x^{\prime}$ and $x$ coordinates, respectively. Let the charge carriers generates in the first layer with the coordinate $x^{\prime}$. We will consider the being found in the second layer drifting in the field SCR charge of $d_{x} \bar{q}$, 


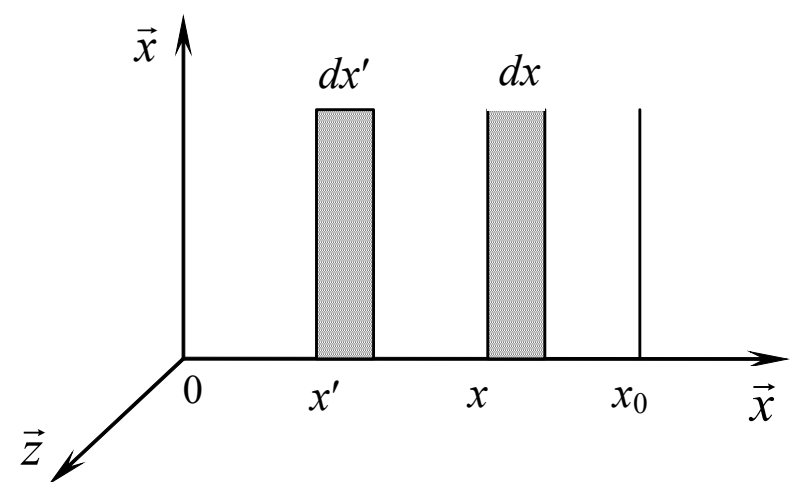

Model of "flat" SCR for estimation of frequency characteristics of photodiode.

obtained by the generation on a unit area in the first layer as responce to radiation falling onto the photodiode crystal.

The drift velocity of this charge is equal to that of components of its charge carriers:

$\vec{v}_{x}=\vec{E}_{x} \bar{\mu}_{x}$

where $\vec{E}_{x}$ is the electric field in a point with the coordinate $x ; \bar{\mu}_{x}$ is the mobility of the considered charge carrier in a point with the coordinate $x$.

We also consider that the electric field is comparatively small, and it is possible to ignore the mobility dependence on the electric field:

$\bar{\mu}_{x}=\bar{\mu}_{0}=\operatorname{const}(x, y, z)$,

where: $\bar{\mu}_{x}=\left\{\begin{array}{l}\bar{\mu}_{x} \text { for holes, } \\ -\bar{\mu}_{n} \text { for electrons, }\end{array}\right.$

and the sign "minus" points to the direction of motion of electrons in the electric field. When the $d_{x} \bar{q}$ charge moves inside the SCR, the electric current $d_{x} I=v_{x} d_{x} q$ flows in the external circuit. From the law of energy conservation, it is possible to write down:

$d_{x} P=d_{x} \vec{F} \cdot \vec{v}_{x}=d_{x} F \cdot v_{x}$,

where $d_{x} P$ is the power expended by the power source on motion of the $d_{x} \bar{q}$ charge; $d_{x} \vec{F}$ is the force affecting the charge in electric field.

Thus, the work (1.6) is the scalar product of the parallel vectors of the force and velocity: $\vec{F} \uparrow \uparrow \vec{v}$.

We note that

$d_{x} P=U_{0} d_{x} I$

where $U_{0}$ is the external applied to SCR voltage equal to the difference of potentials on the SCR scope, $d_{x} \vec{F}=E_{x} d_{x} \vec{q}$,

$\vec{v}_{x}=\vec{i} \frac{d x}{d t}=\frac{d \vec{x}}{d t}$,

$\vec{v}_{x}=\frac{d \vec{x}}{d t}$,

as well as the charge carriers from the first layer into the second one (come behind the time) equal to the time of motion of charge carriers between the layers on the area $\Delta x=\left(x^{\prime}-x_{0}\right)$ that is:

$d_{x} q(t)=d_{x}^{\prime} q\left(t-t^{\prime}\right)$,

where

$t^{\prime}=\int_{x^{\prime}}^{x} \frac{d \vec{x}^{\prime \prime}}{\vec{v}}=\int_{x^{\prime}}^{x} \frac{d \vec{x}^{\prime \prime}}{\vec{E}_{x} \bar{\mu}_{x}} \geq 0$

is the time of the charge motion from the $x^{\prime}$ point to the $x^{\prime \prime}$ one in the considered time point $t$. Then, Eq. (1.7) can be written down as follows:

$U_{0} d_{x} I=d_{x} \vec{F} \cdot \vec{v}_{x}=d_{x} \bar{q} \cdot \vec{E}_{x} \cdot \vec{v}_{x}$

or

$U_{0} d_{x} I(t)=d_{x}^{\prime} \bar{q}\left(t-t^{\prime}\right) \vec{E}_{x}(t) \cdot \vec{v}_{x}(t)$.

The charge $d_{x}^{\prime} \bar{q}\left(t-t^{\prime}\right)$ is created when absorption of radiation by the charge carriers takes place, that is why

$d_{x}^{\prime} \bar{q}\left(t-t^{\prime}\right)=\bar{e}_{0} d_{x}^{\prime} N\left(t-t^{\prime}\right)$,

where $\bar{e}_{0}$ is the charge of one carrier of the considered type with its sign; $d_{x}^{\prime} N\left(t-t^{\prime}\right)$ is the quantity of the electron-hole pairs given rise by the absorbed radiation in the first layer in the time domain $\left(t-t^{\prime} ; t-t^{\prime}+d t\right)$.

It is obvious that

$d_{x}^{\prime} N\left(t-t^{\prime}\right)=\int_{v_{1}} \frac{\gamma(v)}{h v} \cdot \frac{\partial^{2} \Phi\left(t-t^{\prime}\right)}{\partial x^{\prime} \partial v} \cdot d x^{\prime} \cdot d t \cdot d v$,

where $\gamma(v)$ is the quantum yield on the set wavelength; $h v$ is the energy of one absorbed quantum; $\frac{\partial^{2} \Phi\left(t-t^{\prime}\right)}{\partial x^{\prime} \partial v} \cdot d t$ is the energy in the single range of the wave taken in the first layer in the time of $d t ; \Phi\left(t-t^{\prime}\right)$ is the flux of the radiation absorbed in a crystal.

For convenience of calculation, we will take sine similar modulation of radiation for a basis, that is: $\Phi(t)=\bar{\Phi}+\widetilde{\Phi}, \quad \bar{\Phi}=\operatorname{const}(t), \quad \widetilde{\Phi}=\Phi_{0} \sin \omega t$, where $\bar{\Phi}$ is the unvariable (including background) component of the absorbed flux; $\widetilde{\Phi}$ is the variable component of the flux; $\Phi_{0}$ is an amplitude of the variable component in 
the absorbed flux; $\omega t=2 \pi f$ is the modulation frequency of the photoelectric signal (current). follows:

Then, it is possible to write down Eq. (1.14) as

$d_{x}^{\prime} \bar{q}=\bar{e}_{0} \cdot \frac{\partial}{\partial x^{\prime}} \int_{v_{1}} \frac{\gamma(v)}{h v} \cdot \frac{\partial}{\partial v}\left(\bar{\Phi}+\Phi_{0} \sin \omega t\right) d v \cdot d x^{\prime} d t$

and after rearrangements to derive

$d_{x}^{\prime} \bar{q}=d_{x}^{\prime} \bar{q}_{0}+\frac{\partial}{\partial x^{\prime}} I_{0} \cdot \sin \omega t \cdot d x^{\prime} \cdot d t=$

$=d_{x}^{\prime} \bar{q}_{0}+d_{x}^{\prime} \widetilde{q}$

where $I_{0}=\int \frac{\bar{e}_{0} \gamma(v)}{h v} \frac{\partial \Phi_{0}}{\partial v} d v$ is the effective (internal) amplitude of variable by the component of the generated photocurrent; $d_{x}^{\prime} \bar{q}_{0}=\operatorname{const}(t)$.

As the invariable component can be dropped without sacrificing the community of consideration when estimating the frequency properties of the photodetector, further we will consider only the variable component, therefore:

$d_{x}^{\prime} \tilde{q}=\frac{\partial}{\partial x^{\prime}} I_{0} \cdot \sin \omega t \cdot d t \cdot d x^{\prime}=d_{x}^{\prime} I \cdot d t$,

$d_{x} d_{x}^{\prime} I=\frac{1}{U_{0}} \cdot \vec{E}_{x}(x) \cdot \vec{v}_{x}(x) \times$

$\times \frac{\partial}{\partial x^{\prime}} I_{0} \cdot \sin \omega\left(t-t^{\prime}\right) \cdot d t \cdot d x^{\prime}=$

$=\frac{1}{U_{0}} \cdot E_{x}(x) \cdot v_{x}(x) \cdot d t \cdot \sin \omega\left(t-t^{\prime}\right) \cdot \frac{\partial}{\partial x^{\prime}} I_{0} \cdot d x^{\prime}$.

Signs of the vectors in (1.19) vanish due to a parallel $\vec{F}_{x}=\vec{E}_{x} \cdot \bar{e}_{0} \uparrow \uparrow \vec{v}_{x}$, that is scalar product $\vec{E}_{x} \cdot \bar{e}_{0} \cdot \vec{v}_{x}=E_{x} \cdot e_{0} \cdot v_{x}$. Taking (1.10) into account, $d_{x} d_{x}^{\prime} I=\frac{1}{U_{0}} \cdot E_{x}(x) \cdot d x \cdot \sin \omega\left(t-t^{\prime}\right) \times$ $\times \frac{\partial}{\partial x^{\prime}} I_{0} \cdot d x^{\prime}=d_{x}^{\prime} d_{x} I$

Also it is obvious that

$U_{0}=\int_{0}^{x_{0}} E_{x} d x$

In the general case, within the framework of the assumptions accepted by us in relation to the type of modulation of the photoelectric signal, the increase of current in an external circuit (current of photoelectric signal) can be presented as follows: $d_{x} d_{x}^{\prime} I=\frac{E_{x}(x)}{\int_{0}^{x_{0}} E_{x}(x) d x} \times$

$\times \sin \omega\left(t-\int_{x^{\prime}}^{x} \frac{d \vec{x}^{\prime \prime}}{\vec{E}_{x^{\prime \prime}}(x) \bar{\mu}}\right) \cdot \frac{\partial I_{0}}{\partial x^{\prime}} \cdot d x^{\prime} \cdot d x$

Here, the current in the external circuit depends on two functions of coordinates of the electric field and generation density of current of photoelectric signal, and this current value depends on the specific type of these functions.

By the extreme cases being of practical interest for us, there are the cases:

- even strength of the electric field $E_{x}=E_{0}=$ const;

- even density of the volume charge $\nabla E_{x}=\frac{e n}{\varepsilon \varepsilon_{0}}$;

- local, including superficial, generations of current of the photoelectric signal $\frac{\partial I_{0}}{\partial x^{\prime}}=I_{0} \delta\left(x_{0}\right)$, where $\delta\left(x_{0}\right)$ is an ordinary delta function;

- even on the volume of current generation of the photoelectric signal $\frac{\partial I_{0}}{\partial x^{\prime}}=\frac{I_{0}}{x_{0}}$.

Four rearranged functions give four combinations inherent to four really possible situations. Simplicity of the derived expressions make them very comfortable for computations when constructing the photodiodes, and generality of the used presentations provides the high calculation accuracy, confirmed by the subsequent measurements of parameters.

\section{Conclusions}

It was shown that the current in the external circuit depends on two functions (their specific kind) of coordinates of the electric field and generation density of current of photoelectric signal, fully determined by total parameters of material of photodetector and absorbed radiation.

Simplicity, generality and accuracy of the obtained expressions make them suitable for the use to calculate and construct new photodiodes.

\section{References}

1. S.M. Sze, Physics of semiconductor devices, vol. 2. Mir, Moscow, 1984 (in Russian).

2. V.P. Astakhov, D.A. Gindin, V.V. Karpov, K.V. Sorokin, About influence of resistance of surface channel on dark current of quadrant $\mathrm{p}$-i-n photodiodes on silicon // Prikladnaya fizika No 2, p. 79-85 (1999) (in Russian). 
3. Yu.R. Nosov, Photodetectors in optoelectronics // Electronnaya tekhnika, ser. 2, 4(183), p. 33-36 (1986) (in Russian).

4. A.A. Ashcheulov, V.M. Godovanjuk, Yu.G. Dobrovolsky et al., Silicon p-i-n photodiode with little value of dark current // Proc. SPIE 3890, p. 119124 (1999).

5. V.M. Yurgenman, M.A. Trishenkov, Photodiode response kinetics in carrier transit through the space-charge region // Radiotekhnika i elektronika XXII, issue 6, p. 1028 (1977) (in Russian).
6. V.M. Hodovaniouk, I.V. Doktorovych, V.K. Butenko, V.H. Yuryev, Yu.G. Dobrovolsky, Silicon photodiode and preamplifier operation characteristic properties under background radiation conditions // Semiconductor Physics, Quantum Electronics and Optoelectronics 8(1), p. 83-86 (2005).

7. Yu.G. Dobrovolsky, Explosion noise of silicon photodiodes // Fizika i khimiya tverdogo tela 6(2), p. 307-310 (2005) (in Russian). 